\title{
Improvement of glycemic control and reduction of major cardiovascular events in 18 cardiovascular outcome trials: an updated meta-regression
}

\author{
Maria Ida Maiorino ${ }^{* *}$,, Miriam Longo ${ }^{2}$, Lorenzo Scappaticcio ${ }^{2}$, Giuseppe Bellastella ${ }^{2}$, Paolo Chiodini ${ }^{3}$,
} Katherine Esposito ${ }^{1}$ and Dario Giugliano ${ }^{2}$ (D)

\begin{abstract}
Background: Besides providing reassurance about cardiovascular (CV) safety of newer diabetes drugs, cardiovascular outcome trials (CVOTs) have also shown encouraging benefits on some CV endpoints. The contribution of the better glycemic control in the reduction of major cardiovascular events (MACE) remains an open question. The aim of this study is to evaluate the associations between the reduction of HbA1c and risk of MACE, MACE components, hospitalization for heart failure (HF) and all-cause death in CVOTs.
\end{abstract}

Methods: An electronic search up to July 2021 was conducted to determine eligible trials. Systematic review identified eighteen CVOTs reporting prespecified CV outcomes. Pooled summary estimates and $95 \%$ confidence intervals (Cl) were calculated according to the random effects model using the Paule-Mandel method; restricted maximum likelihood estimators were used to estimate model parameters in the metaregression.

Results: The eighteen CVOTs evaluated 161,156 patients and included four trials with dipeptidyl-peptidase-4 inhibitors (DPP-4i), eight trials with glucagon-like peptide-1 receptor agonists (GLP-1RA) and six trials with sodium-glucose cotransporter-2 inhibitors (SGLT-2i). Random-effects model meta-analysis showed an association between treatment and risk of MACE (hazard ratio [HR] 0.90; 95\% Cl 0.86, 0.94, P<0.001), with significant heterogeneity between studies $\left(\mathrm{I}^{2}=45.2 \%\right.$, Q statistic $\left.\mathrm{P}=0.040\right)$. In meta-regression, there was an association between the reduction in $\mathrm{HbA} 1 \mathrm{c}$ at the end of the trial and the HR reduction for MACE (beta $=-0.298, P=0.007)$, with significant heterogeneity $\left(I^{2}=40 \%, Q\right.$ statistic $P=0.04)$; this association was totally driven by the risk reduction of non-fatal stroke, which explained $100 \%$ of between-study variance (beta $=-0.531, R^{2}=100 \%$ ), without heterogeneity $\left(I^{2}=24 \%, Q\right.$ statistic $P=0.206$ ). There was no association between the reduction in $\mathrm{HbA} 1 \mathrm{c}$ and the HR for heart failure or all-cause death.

Conclusions: The reduction of HbA1c in eighteen CVOTs was significantly associated with reduction of non-fatal stroke, explaining all $\left(R^{2}=100 \%\right)$ of the between-study variance. While the contribution of glucose lowering in some CV benefits of newer agents does not influence their indications for the patient with type 2 diabetes, it may hopefully facilitate their use.

*Correspondence: mariaida.maiorino@unicampania.it

${ }^{1}$ Diabetes Unit, Department of Advanced Medical and Surgical Sciences,

University of Campania Luigi Vanvitelli, Naples, Italy

Full list of author information is available at the end of the article

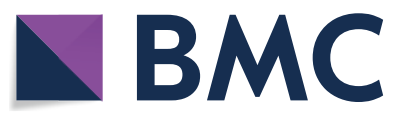

(c) The Author(s) 2021. Open Access This article is licensed under a Creative Commons Attribution 4.0 International License, which permits use, sharing, adaptation, distribution and reproduction in any medium or format, as long as you give appropriate credit to the original author(s) and the source, provide a link to the Creative Commons licence, and indicate if changes were made. The images or other third party material in this article are included in the article's Creative Commons licence, unless indicated otherwise in a credit line to the material. If material is not included in the article's Creative Commons licence and your intended use is not permitted by statutory regulation or exceeds the permitted use, you will need to obtain permission directly from the copyright holder. To view a copy of this licence, visit http://creativecommons.org/licenses/by/4.0/. The Creative Commons Public Domain Dedication waiver (http://creativeco mmons.org/publicdomain/zero/1.0/) applies to the data made available in this article, unless otherwise stated in a credit line to the data. 
Keywords: Cardiovascular outcome trials, Type 2 diabetes, DPP-4i, GLP-1RA, SGLT-2i, Cardiorenal outcomes, Metaregression, MACE, Glycemic control

\section{Introduction}

All the dedicated cardiovascular outcome trials (CVOTs), designed to ensure cardiovascular (CV) safety of the newer glucose-lowering drugs, have provided reassurance about their overall CV safety, and have also shown some encouraging benefits on $\mathrm{CV}$ endpoints. Metaanalyses of CVOTs suggest that glucagon-like peptide-1 receptor agonists (GLP-1RA) and sodium-glucose cotrasporter-2 inhibitors (SGLT-2i) reduce the risk of major adverse cardiovascular events (MACE) to a comparable degree [1-5]. Accordingly, in patients with type 2 diabetes and established $\mathrm{CV}$ disease or multiple $\mathrm{CV}$ risk factors, GLP-1RA or SGLT-2i with demonstrated CV benefits are recommended to reduce the risk of MACE $[6,7]$.

All the CVOTs were designed to reach glycemic equipoise between groups, for minimizing the confounding effect of different glycemic control, and for not exposing participants to the increased risk of microvascular complications, a likely consequence of the suboptimal glycemic control [8]. At the end of CVOTs, however, subjects in the placebo groups had worse glycemic control as compared with those in the treatment groups. In some CVOTs, the imbalance of glycemic control between groups was equal to or greater than that obtained in the UKPDS 34 study [9], the first trial of intensive glycemic control to explore the supposed beneficial effects of near normal hyperglycemia. This imbalance may have masked potential benefits of the glycemic control per se on the CV endpoint. As stated by FDA [10], whether these differences in glycemic control could have contributed directly or indirectly to differences in observed outcomes (i.e., the outcome MACE) is unknown.

The contribution of the better glycemic control in the mediation of the parallel reduction of MACE in CVOTs remains an open question. New diabetes drugs include glucose-lowering agents which were the last to be introduced in the market for the use in people with type 2 diabetes, and specifically both incretins [GLP-1RA and dipeptidyl-peptidase 4 inhibitors-(DPP-4i)] and SGLT2i. The low penetration of these newer drugs in the U.S. market $(7.1 \%$ for both GLP-1RA and SGLT-2i in the last NHANES wave) [11] could also have been a consequence of the lack of enthusiasm of diabetes specialists who are still reluctant to admit that antihyperglycemic drugs may only work independently of blood glucose regulation. A previous meta-analysis with meta-regression of the 15 CVOTs published until 2020 [4] reported a robust association between the reduction in achieved $\mathrm{HbA} 1 \mathrm{c}$ at the end of the trial and the reduction of the risk for MACE, which was driven by the risk reduction of non-fatal stroke only, and apparently restricted to the class of GLP-1RA. The recent availability of three new CVOTs, one involving the SGLT-2i ertuglflozin (VERTIS-CV) [12], one involving the SGLT-1/2 inhibitor sotagliflozin (SCORED) [13], and one involving the long-acting GLP-1RA efpeglenatide (AMPLITUDE-O) [14], offer the opportunity to further disclose whether the improved glycemic control associated with the use of newer glucose-lowering drugs play a role in ameliorating the cardiovascular outlook of people with type 2 diabetes. Therefore, the purpose of this study was to use meta-analysis and meta-regression to investigate the association between improvement of glycemic control, as assessed by reduction of $\mathrm{HbA1c}$ during active treatment, and risk of MACE, MACE components, hospitalization for heart failure (HF) and all-cause death in all CVOTs published to date.

\section{Research design and methods \\ Search strategy and eligibility criteria}

We conducted this systematic review and meta-analysis based on Preferred Reporting Items for Systematic Reviews and Meta-analyses (PRISMA) guidelines [15]. The PRISMA checklist and the protocol are provided in Additional file 1. Neither ethics approval nor patient consent was required for this analysis. The protocol has not been registered on any platform.

Databases searched included PubMed, EMBASE, the Cochrane Central Register of Controlled Trials, the Cochrane Database of Systematic Reviews, and ClinicalTrials.gov (http://www.clinicaltrials.gov). The last search was performed on July 10, 2021. The search terms used were 'dipeptidyl-peptidase inhibitor (DPP-4i)', 'saxagliptin', 'alogliptin', 'sitagliptin', 'linagliptin', 'glucagon-like peptide-1 receptor agonist', 'exenatide,' 'lixisenatide, 'liraglutide', 'semaglutide', 'dulaglutide', 'albiglutide', 'efpeglenatide', 'sodium-glucose co-transporter-2 inhibitor', 'empagliflozin', 'canagliflozin', 'dapagliflozin', 'ertugliflozin', 'sotagliflozin', 'major cardiovascular events', 'MACE, 'myocardial infarction', 'stroke', 'hospitalization for heart failure', 'cardiovascular death' and 'all-cause death. The search was filtered to include only randomized controlled trials (RCTs) or meta-analyses involving humans. Reference lists of prior reviews and meta-analyses were also manually searched to capture other relevant RCTs. 
We included trials if they were RCTs performed in adults with type 2 diabetes, compared add-on therapy with any DPP-4i, GLP-1RA or SGLT-2i against placebo, and included in the outcome (either primary or secondary) MACE, as well as other outcomes required by regulatory agencies for $\mathrm{CV}$ safety studies in diabetes. We excluded trials if they were completed before the FDA guidance of 2008 [16]. Results reported in trial publications were used as the primary source of information. Available additional sources, including, but not limited to, the FDA, European Medicines Agency, and pharmaceutical company websites, were searched to capture any relevant additional data.

\section{Data extraction and quality assessment}

Two investigators (M.I.M. and D.G.) used a standardized tool (provided in Additional file 1) to independently abstract all data. The relevance of studies was assessed with a hierarchical approach based on title, abstract and the full manuscript. After the initial screening of titles and abstracts, the studies included were compared, and any disagreements were resolved by consensus. We evaluated the risk of bias of the included RCTs according to the Cochrane Collaboration's tool for assessing the risk of bias [17].

\section{Outcomes}

The primary outcomes for this meta-analysis were to disclose associations between reductions in $\mathrm{HbA1c}$ and risk of MACE with meta-regression, and to assess the effect of DPP-4i, GLP-1RAs and SGLT-2i on the risk of MACE. Additional preplanned analyses were conducted on the incidence of MACE components ( $\mathrm{CV}$ death, non-fatal myocardial infarction [MI], non-fatal stroke), hospitalization for HF and all-cause death, as well as on associations between reductions in $\mathrm{HbA1c}$ and their risks with meta-regression.

\section{Data synthesis and analysis}

Data were analyzed using Stata version 16.0 (Stata Corporation, College Station, TX, USA). Hazard ratios (HRs) and $95 \%$ confidence intervals $(95 \% \mathrm{CI})$ were collected for CV efficacy outcomes. Heterogeneity between studies was assessed by using the Q statistic and $\mathrm{I}^{2}: \mathrm{I}^{2}<25 \%$ was considered as low in heterogeneity, $\mathrm{I}^{2}>75 \%$ as high in heterogeneity, and a $\mathrm{Q}$ statistic $\mathrm{P}$-value of $<0.10$ was considered significant. Pooled summary estimates and 95\% CI for CV efficacy outcomes were calculated according to a random-effects model using the Paule-Mandel method [18]. Publication bias was assessed visually with funnel plots and with the Egger test [19]. The trim-andfill method [20] was used to estimate the effect of publication bias, if any.
We performed a meta-regression analysis of the CVOTs to describe the relationship between the differences in achieved HbA1c at the end of CVOTs and the corresponding HR for MACE, as well as other CV outcomes. The meta-regression relates the treatment effect to study-level covariates while assuming additivity of within-study and between-studies components of variance. Restricted maximum likelihood estimators were used to estimate model variables [21]. A permutation test (using 1000 reallocations) was used for assessing the true statistical significance of an observed meta-regression finding [22]. Beta on log HR for an absolute change of $1 \%$ in HbA1c was also reported.

\section{Results}

\section{Individual Trial Characteristics}

Of 242 articles screened for eligibility, 18 trials [12-14, 23-37] were eligible and included in the meta-analysis (Additional file 1: Figure S1). There were four trials with DPP-4i, eight trials with GLP-1RA and six trials with SGLT-2i. Their characteristics are summarized in Table 1. The participants were all patients with type 2 diabetes (aged $>18$ years old). All trials were multinational and sponsored by industry. The trials have been published during 2013-2021, with two studies published in 2021. All trials were of parallel-group, double-blind design, and their mean duration ranged from 1.5 to 5.4 years. The baseline $\mathrm{HbA1c}$ level ranged from $7.3 \%(56 \mathrm{mmol} / \mathrm{mol})$ to $8.9 \%(74 \mathrm{mmol} / \mathrm{mol})$ but was almost identical between groups (drug vs. placebo) within the same trial. The populations studied ranged in size from 3183 (PIONEER 6) to 17,160 (DECLARE) and were of similar age (range: 60-69 years).

The 18 CVOTs evaluated 161,156 patients and the following classes of medications: DPP-4i (saxagliptin, alogliptin, sitagliptin, linagliptin) in 43,522 participants; GLP-1RA (lixisenatide, liraglutide, semaglutide, exenatide once-weekly, albiglutide, dulaglutide, oral semaglutide, and efpeglenatide) in 60,080 participants; and SGLT-2is (empagliflozin, canagliflozin, dapagliflozin and sotagliflozin) in 57,554 participants. There was no major risk of bias (Cochrane tool) in any study (Additional file 1: Figure S2). In all trials, MACE was the primary outcome, except CREDENCE and SCORED, in which MACE was a secondary outcome.

\section{MACE}

In the pooled analysis of 18 trials, the risk of MACE was significantly reduced by $10 \%(\mathrm{HR}=0.90,0.86-0.94$, $\mathrm{P}<0.001)$ compared with placebo, with significant heterogeneity between trials $\left(\mathrm{I}^{2}=45.2 \%, \mathrm{P}=0.040\right)$ (Fig. 1 , Additional file 1: Table S1) and evidence of publication bias (Egger test, $\mathrm{P}=0.016$ ). The trim-and-fill method 
Table 1 Key baseline characteristics from each CVOT

\begin{tabular}{|c|c|c|c|c|c|c|}
\hline Trial/year of publication & Study drug/follow-up (y) & Participants (n) & $\begin{array}{l}\text { Mean age } \\
\text { years }\end{array}$ & Baseline HbA1c & Prior CVD (\%) & Study funder \\
\hline \multicolumn{7}{|l|}{ DPP-4i } \\
\hline SAVOR-TIMI 53 & Saxagliptin & 16,492 & 65.0 & $8.0 \%$ & 78.6 & AstraZeneca \\
\hline 2013 & 2.1 & & & $64 \mathrm{mmol}$ & & Bristol-Myers \\
\hline EXAMINE & Alogliptin & 5380 & 61.0 & $8.0 \%$ & 100 & Takeda \\
\hline 2013 & 1.5 & & & $64 \mathrm{mmol}$ & & \\
\hline TECOS & Sitagliptin & 14,6671 & 66.0 & $7.3 \%$ & 74 & Merck Sharp \& \\
\hline 2015 & 2.8 & & & $56 \mathrm{mmol}$ & & Dohme \\
\hline CARMELINA & Linagliptin & 6979 & 65.9 & $7.9 \%$ & 100 & Boehringer \\
\hline 2018 & 2.2 & & & $63 \mathrm{mmol}$ & & Ingelheim \\
\hline \multicolumn{7}{|l|}{ GLP-1RA } \\
\hline ELIXA & Lixisenatide & 6068 & 60 & $7.7 \%$ & 100 & Sanofi \\
\hline 2015 & 2.1 & & & $61 \mathrm{mmol}$ & & \\
\hline LEADER & Liraglutide & 9340 & 64.3 & $8.7 \%$ & 72.4 & Novo-Nordisk \\
\hline 2016 & 3.8 & & & $72 \mathrm{mmol}$ & & \\
\hline SUSTAIN-6 & Semaglutide & 3297 & 64.6 & $8.7 \%$ & 83.0 & Novo-Nordisk \\
\hline 2016 & 3.1 & & & $72 \mathrm{mmol}$ & & \\
\hline EXSCEL & Exenatide OW & 14,752 & 62.0 & $8.0 \%$ & 73.1 & Amylin Pharma- \\
\hline 2017 & 3.2 & & & $64 \mathrm{mmol}$ & & ceuticals \\
\hline HARMONY & Albiglutide & 9463 & 64.0 & $8.7 \%$ & 100 & GlaxoSmithKline \\
\hline 2018 & 1.6 & & & $72 \mathrm{mmol}$ & & \\
\hline REWIND & Dulaglutide & 9901 & 66.2 & $7.2 \%$ & 31.4 & Boehringer/Lilly \\
\hline 2019 & 5.4 & & & $55 \mathrm{mmol}$ & & \\
\hline PIONEER 6 & Semaglutide $O$ & 3183 & 66.0 & $8.2 \%$ & 84.7 & Novo-Nordisk \\
\hline 2019 & 1.3 & & & $66 \mathrm{mmol}$ & & \\
\hline AMPLITUDE-0 & Efpeglenatide & 4076 & 64.5 & $8.9 \%$ & 89.6 & Sanofi \\
\hline 2021 & 1.8 & & & $74 \mathrm{mmol}$ & & \\
\hline \multicolumn{7}{|l|}{ SGLT-2i } \\
\hline EMPA-REG & Empagliflozin & 7021 & 63.2 & $8.1 \%$ & 100 & Boehringer/Lilly \\
\hline 2015 & 3.1 & & & $65 \mathrm{mmol}$ & & \\
\hline CANVAS & Canagliflozin & 10,142 & 63.2 & $8.2 \%$ & 72.2 & Janssen \\
\hline 2017 & 2.4 & & & $66 \mathrm{mmol}$ & & \\
\hline DECLARE & Dapagliflozin & 17,160 & 63.8 & $8.3 \%$ & 40.6 & AstraZeneca \\
\hline 2017 & 4.2 & & & $67 \mathrm{mmol}$ & & \\
\hline CREDENCE & Canagliflozin & 4401 & 63.0 & $8.3 \%$ & 50.4 & Janssen \\
\hline 2019 & 2.6 & & & $67 \mathrm{mmol}$ & & \\
\hline VERTIS-CV & Ertugliflozin & 8246 & 64.4 & $8.2 \%$ & 100 & Merck Sharp \& \\
\hline 2020 & 3.0 & & & $66 \mathrm{mmol}$ & & Dohme \\
\hline SCORED & Sotagliflozin & 10,584 & 69.0 & $8.3 \%$ & 48.6 & Sanofi/Lexicon \\
\hline 2021 & 1.4 & & & $67 \mathrm{mmol}$ & & Pharmaceuticals \\
\hline
\end{tabular}

OW once weekly, $O$ oral

indicated that this publication bias did not change the statistical significance of the estimate (HR 0.91, 95\% CI 0.87-0.96). Meta-regression showed an association (Fig. 2) between the reduction in achieved HbA1c at the end of the trial and the HR reduction for MACE (beta $=-0.298, P=0.007)$, explaining almost all (97\%) of the between-study variance (Table 2). For every $1 \%$
(10.93 $\mathrm{mmol} / \mathrm{mol})$ greater average reduction in $\mathrm{HbA} 1 \mathrm{c}$, the risk of MACE decreased by $26 \%$. Compared with placebo, DPP-4i showed a neutral effect on MACE, while the use of both GLP-1RA and SGLT-2i was associated with significant reductions in MACE (14\% and $11 \%$, respectively), with non-significant heterogeneity for SGLT-2i (Fig. 1, Additional file 1: Table S1). The trials that showed 


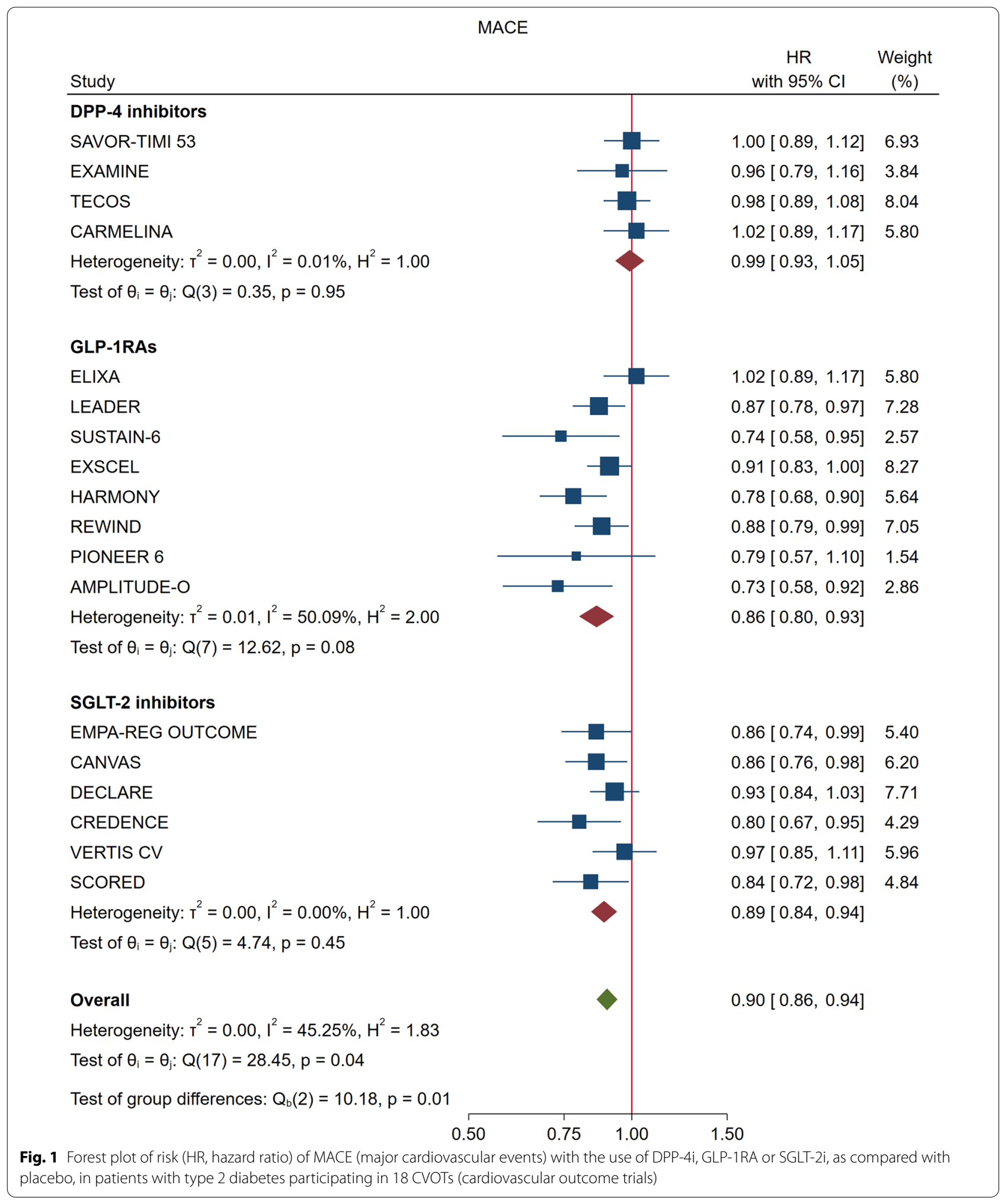

a significant benefit on MACE were LEADER, SUSTAIN 6, HARMONY, REWIND and AMPLITUDE-O for GLP-1RA, and EMPA-REG OUTCOME, CANVAS, CREDENCE, and SCORED for SGLT-2i (Fig. 1). 

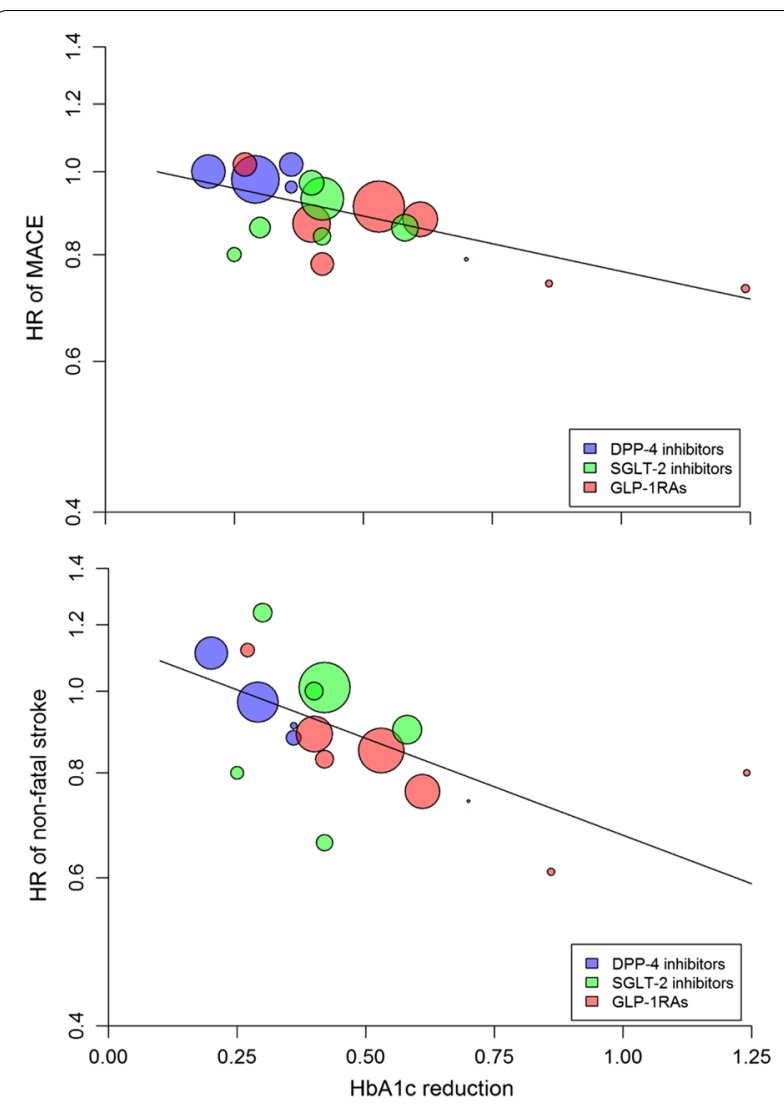

Fig. 2 Meta-regression analysis between the differences

(intervention minus placebo) in achieved $\mathrm{HbA} 1 \mathrm{c}$ at the end of CVOTs and the corresponding hazard ratio (HR) for MACE (top) or non-fatal stroke (bottom) in patients with type 2 diabetes participating in 18 CVOTs

Table 2 Values of heterogeneity and Beta on $\log H R$ in the meta-regression

\begin{tabular}{lccccc}
\hline & $\mathbf{I}^{\mathbf{2}}$ & $\begin{array}{l}\text { P-value of } \\
\text { heterogeneity }\end{array}$ & $\begin{array}{l}\text { Beta on log } \\
\text { (HR) }\end{array}$ & P-value & $\mathbf{R}^{\mathbf{2}}$ \\
\hline MACE & $40 \%$ & 0.040 & -0.298 & 0.007 & $97 \%$ \\
CV death & $39 \%$ & 0.047 & -0.176 & 0.311 & $4 \%$ \\
Non-fatal MI & $30 \%$ & 0.108 & -0.181 & 0.256 & $3 \%$ \\
$\begin{array}{l}\text { Non-fatal } \\
\text { stroke }\end{array}$ & $21 \%$ & 0.206 & -0.531 & 0.008 & $100 \%$ \\
$\begin{array}{l}\text { Heart failure } \\
\text { All-cause }\end{array}$ & $70 \%$ & $<0.001$ & -0.186 & 0.474 & $0 \%$ \\
death & $48 \%$ & 0.012 & -0.196 & 0.192 & $24 \%$ \\
\hline
\end{tabular}

\section{Cardiovascular death}

There was a significant $(\mathrm{P}<0.001) 12 \%$ risk reduction in CV death associated with the use of newer drugs compared with placebo, with significant heterogeneity $\left(\mathrm{I}^{2}=39 \%, \mathrm{P}=0.047\right)$ (Table 2$)$ and evidence of publication bias (Egger test, $\mathrm{P}=0.028$ ). However, the trim-andfill method indicated that this publication bias did not impact the estimate. The association between the reduction in achieved $\mathrm{HbA} 1 \mathrm{c}$ and $\mathrm{CV}$ death was not significant (beta $=-0.176, \mathrm{P}=0.311$, variance explained $=4 \%$ ) (Fig. 3, Table 2). The use of both GLP-1RA and SGLT-2i was associated with a significant $13 \%$ and $16 \%$ risk reduction in $\mathrm{CV}$ death, respectively, with non-significant heterogeneity for GLP-1RA (Additional file 1: Table S1). The trials that showed a clear significant benefit on $\mathrm{CV}$ death were LEADER and PIONEER 6 for GLP-1RA, and EMPA-REG OUTCOME for SGLT-2i.

\section{Non-fatal myocardial infarction}

Overall, there was a significant $(\mathrm{P}=0.007) 8 \%$ risk reduction of non-fatal MI associated with the use of newer drugs compared with placebo, with non-significant heterogeneity $\left(\mathrm{I}^{2}=36.5 \%, \mathrm{P}=0.108\right)$ (Additional file 1 : Table S1) and no evidence of publication bias (Egger test, $\mathrm{P}=0.364)$. The association between the reduction in achieved HbA1c and nonfatal MI was not significant (beta $=-0.181, \quad \mathrm{P}=0.256, \quad$ variance explained $=3 \%$ ) (Fig. 4, Table 2). The use of both GLP-1RA and SGLT-2i was associated with a significant $9 \%$ and $13 \%$ risk reduction in CV death, respectively, with non-significant heterogeneity for both (Additional file 1: Table S1). No single trial in any class produced a significant benefit on nonfatal MI, except for albiglutide (HARMONY trial) and sotagliflozin (SCORED trial).

\section{Non-fatal stroke}

There was a significant $(\mathrm{P}=0.012) 9 \%$ risk reduction of non-fatal stroke associated with the use of newer

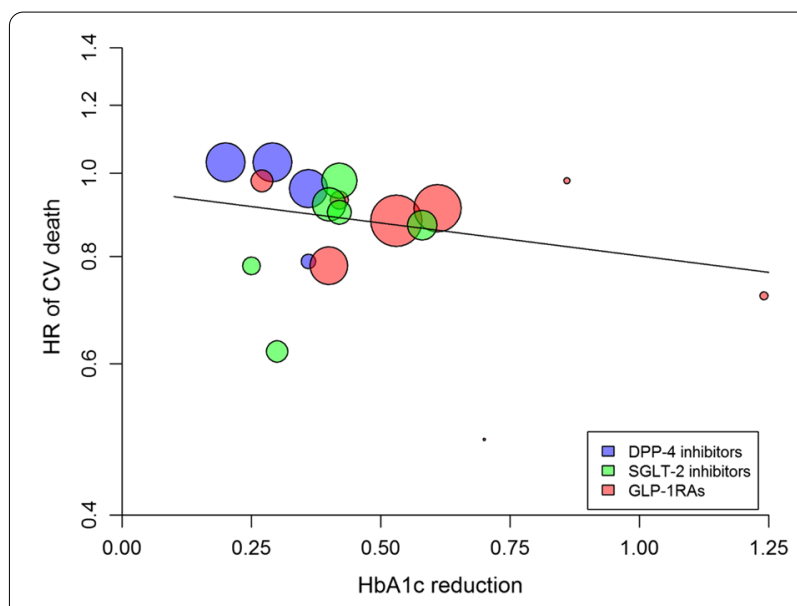

Fig. 3 Meta-regression analysis between the differences in achieved $\mathrm{HbA1c}$ at the end of cardiovascular outcome trials (CVOTs) and the corresponding hazard ratio (HR) for CV death in patients with type 2 diabetes participating in 18 CVOTs 


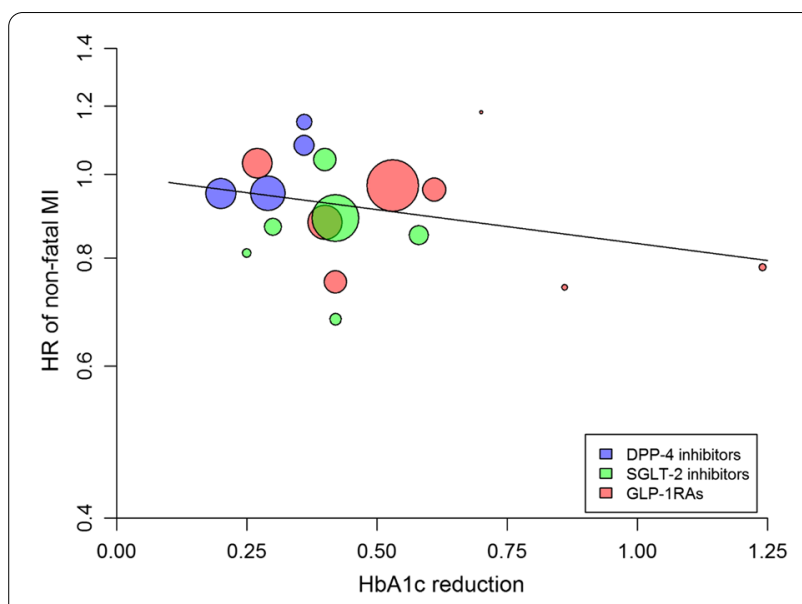

Fig. 4 Meta-regression analysis between the differences in achieved $\mathrm{HbA1c}$ at the end of cardiovascular outcome trials (CVOTs) and the corresponding hazard ratio (HR) for non-fatal Ml in patients with type 2 diabetes participating in 18 CVOTs

drugs, which was largely driven by the $16 \%$ reduction associated with the use of GLP-1RA (Additional file 1: Table S1), with no significant heterogeneity $\left(\mathrm{I}^{2}=21.3 \%\right.$, $\mathrm{P}=0.206$ ) and no evidence of publication bias (Egger test, $\mathrm{P}=0.233$ ). The association between the reduction in achieved HbAlc and non-fatal stroke was highly significant (beta $=-0.531, \mathrm{P}=0.008$, variance explained $=100 \%$ ) (Table 2 ) and accounted for almost all the association between MACE and $\mathrm{HbAlc}$ reduction. For every $1 \%(10.93 \mathrm{mmol} / \mathrm{mol})$ greater average reduction in HbA1c, the risk of non-fatal stroke decreased by $41 \%$. The trials that showed a clear significant benefit on non-fatal stroke were SUSTAIN-6 and REWIND for GLP-1RA and SCORED for SGLT-2i.

\section{Hospitalization for HF}

In pooled analysis of the 18 trials, the risk of hospitalization for HF showed a significant $(\mathrm{P}<0.001) 16 \%$ reduction with the newer antihyperglycemic drugs, with significant heterogeneity between trials $\left(\mathrm{I}^{2}=69.2 \%\right.$, $\mathrm{P}<0.001$ ) (Additional file 1: Table S1), and no evidence of publication bias (Egger test, $\mathrm{P}=0.413$ ). There was no association between the reduction in achieved HbA1c and the HR for HF (beta $=-0.186, \mathrm{P}=0.474$, variance explained $=0 \%$ ) (Fig. 5, Table 2). The use of both GLP1RA and SGLT-2 was associated with significant reductions of the HF risk (10\% and $32 \%$, respectively), with null heterogeneity (Additional file 1: Table S1). All SGLT-2i reduced the risk of HF; among GLP-1RA, efpeglenatide only did so (Fig. 5).

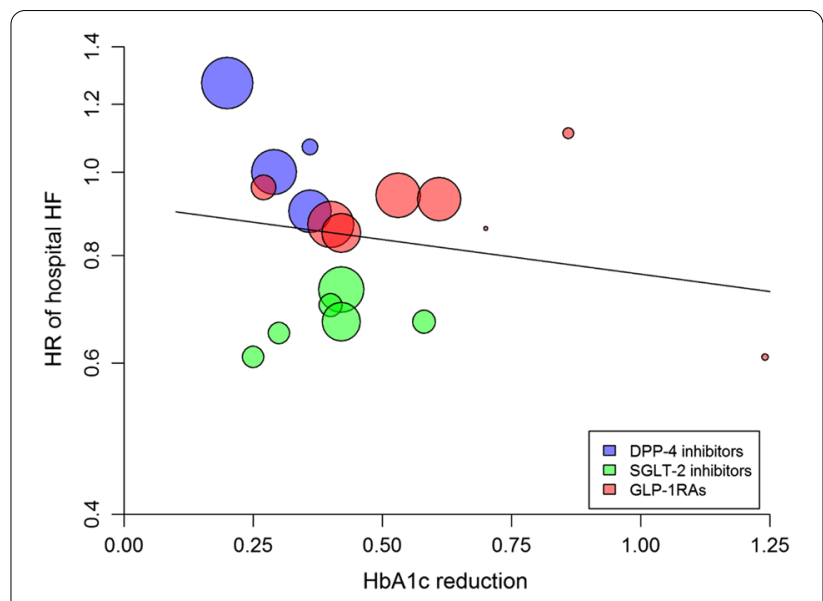

Fig. 5 Meta-regression analysis between the differences in achieved $\mathrm{HbA} 1 \mathrm{C}$ at the end of cardiovascular outcome trials (CVOTs) and the corresponding hazard ratio (HR) for hospitalization for heart failure (HF) in patients with type 2 diabetes participating in 18 CVOTs

\section{All-cause death}

Overall, there was a significant $(\mathrm{P}=0.001) 9 \%$ risk reduction of all-cause death associated with the use of newer drugs compared with placebo, with significant heterogeneity $\left(\mathrm{I}^{2}=55.6 \%, \mathrm{P}=0.012\right)$ (Additional file 1 : Table S1) and some evidence of publication bias (Egger test, $\mathrm{P}=0.074)$. However, the trim-and-fill method indicated that this publication bias did not impact the estimate. There was a nonsignificant association between the reduction in achieved HbA1c and the HR for all-cause death (beta $=-0.196, \mathrm{P}=0.192$, variance explained $=24 \%$ ) (Fig. 6, Table 2). The use of both GLP1RA and SGLT-2i was associated with a significant $12 \%$

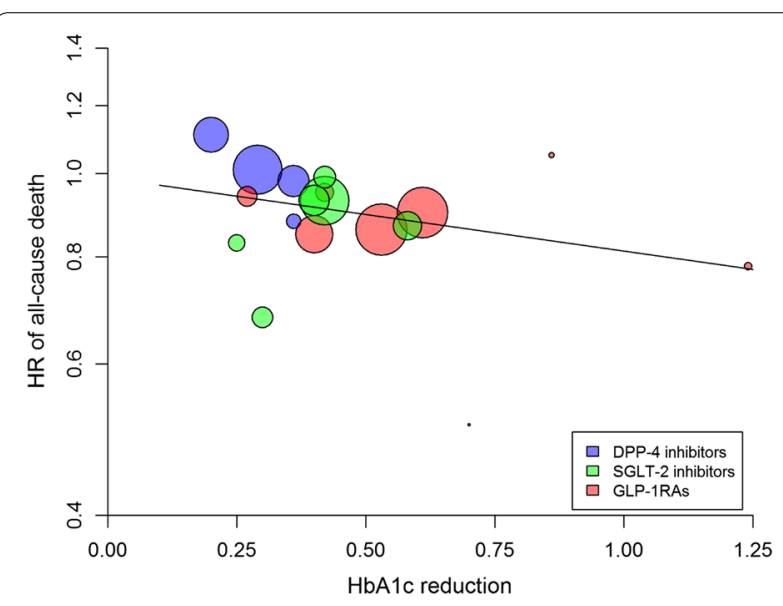

Fig. 6 Meta-regression analysis between the differences in achieved $\mathrm{HbA1c}$ at the end of CVOTs and the corresponding hazard ratio (HR) for all-cause death in patients with type 2 diabetes participating in 18 CVOTs 
and $13 \%$ risk reduction in all-cause death, respectively, with a significant heterogeneity for SGLT-2i (Additional file 1: Table S1). The trials that showed a significant benefit on all-cause death were LEADER, EXSCEL and PIONEER 6 for GLP-1RA and EMPA-REG OUTCOME for SGLT-2i.

\section{Discussion}

This analysis used the tools of meta-analysis and metaregression to explore the association between changes in HbA1c observed in the treatment arms of CVOTs, as compared to the placebo arms, and the reduction of MACE. The results of the meta-regression analysis of the 18 CVOTs in 161,156 patients with type 2 diabetes show that the reduction of $\mathrm{HbA} 1 \mathrm{c}$ during treatment with DPP-4i, GLP-1RA or SGLT-2i is associated with reduction of MACE, explaining almost all $\left(R^{2}=97 \%\right)$ of the between-study variance. The risk reduction of MACE was almost completely driven by the reduction of nonfatal stroke, whose association explains $100 \%$ of betweenstudy variance, and is unique in holding this relationship among MACE components. In the pooled analysis of the 18 CVOTs, we found a $10 \%$ reduction of MACE risk in patients treated with the newer antihyperglycemic drugs, which remained significant for GLP-1RA and SGLT-2i (14\% and $11 \%$ risk reduction, respectively), and was null for DPP-4i. The inclusion of VERTIS, SCORED and AMPLITUDE-O trials in the qualitative synthesis confirm and extend results coming from our previous meta-analysis [4], corroborating the robustness of these findings.

To minimize the confounding effect of differences in glycemic control, all the CVOTs were designed to promote glycemic equipoise between the two arms (intervention versus placebo) of each trial. However, it was not mandatory that glycemic equipoise was a prerequisite for the completion of the trial; therefore, participants in the placebo group had worse glycemic control as compared with those in the treatment group. Although intensive glycemic control has an imperfect role in reducing the CV burden of patients with type 2 diabetes, it can reduce the risk of MACE by $9 \%$ [38], which is not quite dissimilar to the overall reduction of MACE (10\%) found in the present meta-analysis. Moreover, a meta-regression analysis of randomized controlled trials comparing intensive blood glucose control obtained with conventional diabetes therapy (mainly insulin) with a less intensive regimen showed limited benefit of intensive glycemic control in people with type 2 diabetes and myocardial infarction, with a significant risk of serious hypoglycemia [39]. In general, the CVOTs that obtained the best glycemic equipoise between treatments presented a smallest effect on MACE. Accordingly, DPP-4i produced the least reduction of $\mathrm{HbA1c}$ (mean reduction $=-0.30 \%$ ) and the least effect on MACE (HR, 0.99). On the other hand, the relation does not entirely apply for SGLT-2i because, at the almost same level of $\mathrm{HbA} 1 \mathrm{c}$ reduction (mean reduction $=-0.39 \%$ ), the risk of MACE was reduced by $11 \%$ $(\mathrm{HR}=0.89)$, suggesting for SGLT-2i additional CV benefits independent of glucose regulation. The mean $\mathrm{HbA1c}$ reduction obtained in the eight CVOTs with GLP-1RA was greater (mean $\mathrm{HbA} 1 \mathrm{c}$ reduction $=-0.63 \%$ ) than that obtained with DPP-4i or SGLT-2i and was associated with a greater reduction of MACE risk.

The association between reduction in HbA1c levels by newer drugs and the risk of MACE was entirely driven by non-fatal stroke, suggesting that blood glucose reduction may play a more important role than previously thought in reducing the risk of non-fatal stroke during treatment with the newer glucose-lowering drugs. Reduced risk of hypoglycemia observed with these drugs may also have played a role, as severe hypoglycemic episodes within the previous 3 months were associated with increased risk for MACE in the Veterans Affairs Diabetes Trial [40]. With this perspective, GLP-1RA may be considered in patients with type 2 diabetes and a previous stroke or those at high risk of stroke [41]. On the other hand, the lack of the association between $\mathrm{HbA} 1 \mathrm{c}$ reduction and the risk of other atherosclerosis-based conditions, including myocardial infarction, deserves further investigation. The higher prevalence of an established coronary heart diseases (nearly $60 \%$ in the global population included in the trials), which can predispose to a greater risk of new events, may have played a role in blunting the potential favorable effects of blood glucose reduction with newer glucose-lowering drugs on the heart.

Diabetes continues to be a relevant cause of disability worldwide, despite the availability of glucose-lowering drugs which have proved to be protective against major cardiovascular events in people at high risk of vascular complications [42, 43]. The residual cardiorenal risk after successful glycemic control with new glucose-lowering agents is similar for GLP-1RA and SGLT-2i and null for DPP-4i for MACE [44]. SGLT-2i, as compared with GLP$1 R A$, removed more risk for both kidney outcomes and HF, whereas DPP-4i have no clinically important benefits on these outcomes [44]. Moreover, beyond their glucoselowering effects, these drugs have several pleiotropic protective properties, which include anti-inflammatory and immunomodulatory activities, antifibrotic and antithrombotic effects, and vascular endothelial protective properties which may be responsible for their potential favorable impact on clinical outcomes in diabetic people with COVID-19 $[5,45]$. There is also evidence from observational studies that DPP-4i decrease the risk for COVID-19-related death [46, 47]. 
This study has limitations. Meta-regression evaluates mean data that do not necessarily correspond to individual patient data. In order to minimize the risk of ecological fallacy, our results should therefore be considered as exploratory, and thus always interpreted in conjunction with the effect from the subset of studies most relevant to the patients. This study has also strengths. The number of studies included in the meta-regression is higher than the minimum of 10 recommended by the Cochrane Collaboration Handbook [48]; moreover, all the CVOTs were powered to assess MACE outcome.

\section{Conclusions}

In conclusion, blood glucose lowering may play a role in reducing the risk of MACE during treatment with the newer agents. However, this mediation is highly significant for non-fatal stroke only; the other two components of MACE (non-fatal MI and CV death), as well as HF and all-cause death, are not associated with improvement of glycemic control during treatment. The possible contribution of glucose lowering in mediating part of CV benefit on MACE by GLP-1RA or SGLT-2i does not influence their indications for the patient with type 2 diabetes. Current recommendations by ADA [6] state that GLP1RA should be considered in patients with established, or at a high risk of CV disease, and SGLT-2i for patients with heart failure or chronic kidney disease. Acknowledging the potential contributive role of glucose lowering in decreasing, at least in part, MACE risk of patients with type 2 diabetes may hopefully overcome some residual reluctance of clinicians who still prefer older diabetes drugs without a clear evidence of CV protection.

\section{Abbreviations}

ADA: American Diabetes Association; CV: Cardiovascular; CVOTs: Cardiovascular outcome trials; GLP-1RA: Glucagon-like peptide-1 receptor agonists; HR: Hazard ratios; MACE: Major cardiovascular events; SGLT-2i: Sodium-glucose transporter-2 inhibitors; T2DM: Type 2 diabetes mellitus.

\section{Supplementary Information}

The online version contains supplementary material available at https://doi. org/10.1186/s12933-021-01401-8.

Additional file 1. Additional figure and table.

\section{Acknowledgements}

Not applicable.

\section{Authors' contributions}

DG and KE participated in the study concept and design. GB, ML, LS and MIM collected data. PC and DG. carried out the statistical analyses. All authors contributed to drafting of the manuscript, had full access to all the data in the study, and had final decision to submit for publication. All authors read and approved the final manuscript.

Funding

None.

Availability of data and materials

All data generated or analyzed during this study are included in this published article and in its Additional file.

\section{Declarations}

Ethics approval and consent to participate

Not applicable.

Consent for publication

Not applicable.

\section{Competing interests}

M. I. M. has held lectures for Astrazeneca, Sanofi, NovoNordisk, and MSD. K. E. received a consultancy fee from Eli Lilly and has held lectures for Eli Lilly, Sanofi, and Novo Nordisk. D. G. received a consultancy fee from Eli Lilly and has held lectures for Eli Lilly, Boehringer Ingelheim, Novo Nordisk, Novartis, Mundipharma, Sanofi. No other potential conflicts of interest relevant to this article were reported.

\section{Author details}

${ }^{1}$ Diabetes Unit, Department of Advanced Medical and Surgical Sciences, University of Campania Luigi Vanvitelli, Naples, Italy. ${ }^{2}$ Division of Endocrinology and Metabolic Diseases, Department of Advanced Medical and Surgical Sciences, University of Campania Luigi Vanvitelli, Naples, Italy. ${ }^{3}$ Medical Statistics Unit, University of Campania Luigi Vanvitelli, Naples, Italy.

Received: 19 August 2021 Accepted: 9 October 2021

Published online: 18 October 2021

\section{References}

1. Zelniker TA, Wiviott SD, Raz I, et al. Comparison of the effects of glucagonlike peptide receptor agonists and sodium-glucose cotransporter 2 inhibitors for prevention of major adverse cardiovascular and renal outcomes in type 2 diabetes mellitus. Circulation. 2019;139:2022-31.

2. Giugliano D, Maiorino Ml, Longo M, et al. Type 2 diabetes and risk of heart failure: a systematic review and meta-analysis from cardiovascular outcome trials. Endocrine. 2019;65:15-24.

3. Giugliano D, Longo M, Maiorino Ml, et al. Efficacy of SGLT-2 inhibitors in older adults with diabetes: Systematic review with meta-analysis of cardiovascular outcome trials. Diab Res Clin Pr. 2020;162:108114.

4. Giugliano D, Bellastella G, Longo M, Scappaticcio L, Maiorino MI, Chiodini P, Esposito K. Relationship between improvement of glycaemic control and reduction of major cardiovascular events in 15 cardiovascular outcome trials: a meta-analysis with meta-regression. Diabetes Obes Metab. 2020;22:1397-405.

5. Longo M, Caruso P, Maiorino Ml, et al. Treating type 2 diabetes in COVID19 patients: the potential benefits of injective therapies. Cardiovasc Diabetol. 2020;19:115.

6. American Diabetes Association. Standards of Medical Care in Diabetes-2021 Pharmacologic approaches to glycemic treatment Diabetes. Care. 2021;44(Suppl 1):S111-24.

7. Cosentino F, Grant PJ, Victor Aboyans V, et al. 2019 ESC Guidelines on diabetes, pre-diabetes, and cardiovascular diseases developed in collaboration with the EASD. Eur Heart J. 2020;41:255-323.

8. Taylor SI, Leslie BR. Cardiovascular outcome trials of diabetes drugs: lessons learned. J Clin Invest. 2018;128:893-6.

9. UK Prospective Diabetes Study (UKPDS) Group. Effect of intensive bloodglucose control with metformin on complications in overweight patients with type 2 diabetes (UKPDS 34). Lancet. 1998;352:854-865. 
10. FDA. Endocrinologic and Metabolic Drug Advisory Committee (EMPAREG). 2016. https://www.fda.gov/media/98910/download. Accessed 25 July 2021.

11. Fang M, Wang D, Coresh J, Selvin E. Trends in diabetes treatment and control in U.S. adults, 1999-2018. N Engl J Med. 2021;384:2219-2228.

12. Cannon CP, Pratley R, Dagogo-Jack S, et al, for the VERTIS CV investigators. Cardiovascular outcomes with ertugliflozin in type 2 diabetes. N Engl J Med. 2020;383:1425-1435.

13. Bhatt DL, Szarek M, Pitt B, Cannon CP, et al; for the SCORED investigators. Sotagliflozin in patients with diabetes and chronic kidney disease. N Engl J Med. 2021;384:129-139.

14. Gerstein HC, Sattar N, Rosenstock J, et al; for the AMPLITUDE-O Trial Investigators. Cardiovascular and renal outcomes with efpeglenatide in type 2 diabetes. New Engl J Med. 2021. https://doi.org/10.1056/NEJMoa2108269

15. Liberati A, Altman DG, Tetzlaff J, et al. The PRISMA statement for reporting systematic reviews and meta-analyses of studies that evaluate health care interventions: explanation and elaboration. Ann Intern Med 2009;151:W65-94

16. Food and Drug Administration. Guidance for industry: diabetes mellitus - evaluating cardiovascular risk in new antidiabetic therapies to treat type 2 diabetes. Silver Spring, Maryland: Food and Drug Administration, December 2008. www.fda.gov/downloads/Drugs/Guidances/ucm07 1627.pdf. Accessed 24 July 2021

17. Higgins JP, Altman DG, Gøtzsche PC, et al. The Cochrane Collaboration's tool for assessing risk of bias in randomised trials. Br Med J. 2011;343:d5928

18. Langan D, Higgins JPT, Simmonds M. Comparative performance of heterogeneity variance estimators in meta-analysis: a review of simulation studies. Res Synth Methods. 2017;8:181-98.

19. Egger M, Smith GD, Schneider M, Minder C. Bias in metaanalysis detected by a simple, graphical test. Br Med J. 1997:315:629-34.

20. Duval SJ, Tweedie RL. A non-parametric "trim and fill" method of accounting for publication bias in meta-analysis. J Am Stat Ass. 2000;95:89-98.

21. Van Houwelingen HC, Arends LR, Stijnen T. Advanced methods in meta-analysis: multivariate approach and meta-regression. Stat Med. 2002;21:589-624.

22. Higgins JP, Thompson SG. Controlling the risk of spurious findings from metaregression. Stat Med. 2004;23:1663-82.

23. Scirica BM, Bhatt DL, Braunwald E, et al. SAVOR-TIMI 53 steering committee and investigators Saxagliptin and cardiovascular outcomes in patients with type 2 diabetes mellitus. N Engl J Med. 2013;369:1317-26.

24. White WB, Cannon CP, Heller SR, et al. Alogliptin after acute coronary syndrome in patients with type 2 diabetes. N Engl J Med. 2013;369:1327-35.

25. Green JB, Bethel MA, Armstrong PW, et al. Effect of sitagliptin on cardiovascular outcomes in type 2 diabetes. N Engl J Med. 2015;373:232-42.

26. Rosenstock J, Perkovic V, Johansen OE, et al. Effect of linagliptin vs placebo on major cardiovascular events in adults with type 2 diabetes and high cardiovascular and renal risk. The CARMELINA randomized clinical trial. JAMA. 2019:321:69-79.

27. Pfeffer MA, Claggett B, Diaz R, et al. Lixisenatide in patients with type 2 diabetes and acute coronary syndrome. N Engl J Med. 2015;373:2247-57.

28. Marso SP, Daniels GH, Brown-Frandsen K, et al. Liraglutide and cardiovascular outcomes in type 2 diabetes. N Engl J Med. 2016;375:311-22.

29. Marso SP, Bain SC, Consoli A, et al. Semaglutide and cardiovascular outcomes in patients with type 2 diabetes. N Engl J Med. 2016;375:1834-44.

30. Holman RR, Bethel MA, Mentz RJ, et al. Effects of once-weekly exenatide on cardiovascular outcomes in type 2 diabetes. $N$ Engl J Med. 2017:377:1228-39.

31. Hernandez AF, Green JB, Janmohamed S, et al. Albiglutide and cardiovascular outcomes in patients with type 2 diabetes and cardiovascula disease (Harmony outcomes): a double-blind, randomized placebocontrolled trial. Lancet. 2018:392:1519-29.
32. Gerstein HC, Colhoun HM, Dagenais GR, et al. Dulaglutide and cardiovascular outcomes in type 2 diabetes (REWIND): a doubleblind, randomised placebo-controlled trial. Lancet. 2019:394:121-30.

33. Husain M, Birkenfeld AL, Donsmark M, et al; for the PIONEER 6 investigators. Oral semaglutide and cardiovascular outcomes in patients with type 2 diabetes. N Engl J Med. 2019;381:841-851.

34. Zinman B, Wanner C, Lachin JM, et al. Empagliflozin, cardiovascular outcomes, and mortality in type 2 diabetes. N Engl J Med. 2015;373:2117-28.

35. Neal B, Perkovic V, Mahaffey KW, et al. Canagliflozin and cardiovascular and renal events in type 2 diabetes. N Engl J Med. 2017;377:644-57.

36. Wiviott SD, Raz I, Bonaca MP, et al. Dapagliflozin and cardiovascular outcomes in type 2 diabetes. N Engl J Med. 2019;380:347-57.

37. Perkovic V, Jardine MJ, Neal B, et al. Canagliflozin and renal outcomes in type 2 diabetes and nephropathy. N Engl J Med. 2019;380:2295-306.

38. Giugliano D, Maiorino MI, Bellastella G, Chiodini P, Esposito K. Glycemic control, preexisting cardiovascular disease, and risk of major cardiovascular events in patients with type 2 diabetes mellitus: systematic review with meta-analysis of cardiovascular outcome trials and intensive glucose control trials. J Am Heart Assoc. 2019;8:e012356.

39. Chatterjee S, Sharma A, Lichstein E, Mukherjee D. Intensive glucose control in diabetics with an acute myocardial infarction does not improve mortality and increases risk of hypoglycemia-a meta-regression analysis. Curr Vasc Pharmacol. 2013;11(1):100-4.

40. Davis SN, Duckworth W, Emanuele N, et al. Effects of severe hypoglycemia on cardiovascular outcomes and death in the veterans affairs diabetes trial. Diabetes Care. 2019;42:157-63.

41. Bellastella G, Maiorino MI, Longo M, et al. Glucagon-like peptide-1 receptor agonists and prevention of stroke. Systematic review of cardiovascular outcome trials with meta-analysis. Stroke. 2020;51:666-9.

42. Carlsson S, Andersson T, Talbäck M, Feychting M. Mortality rates and cardiovascular disease burden in type 2 diabetes by occupation, results from all Swedish employees in 2002-2015. Cardiovasc Diabetol. 2021;20(1):129

43. Cha JJ, Kim H, Ko YG, et al; K-VIS (Korean Vascular Intervention Society) investigators. Influence of preprocedural glycemic control on clinical outcomes of endovascular therapy in diabetic patients with lower extremity artery disease: an analysis from a Korean multicenter retrospective registry cohort. Cardiovasc Diabetol. 2020;19(1):97.

44. Giugliano D, Maiorino MI, Bellastella G, Esposito K. The residual cardiorenal risk in type 2 diabetes. Cardiovasc Diabetol. 2021;20(1):36.

45. Han T, Ma S, Sun C, et L. The Association Between Anti-diabetic Agents and Clinical Outcomes of COVID-19 in Patients with Diabetes: A Systematic Review and Meta-Analysis. Arch Med Res. 2021:S0188-4409(21)00167-3.

46. Patoulias D, Doumas M. Dipeptidyl peptidase-4 inhibitors and COVID19-related deaths among patients with type 2 diabetes mellitus: a meta-analysis of observational studies. Endocrinol Metab (Seoul). 2021;36(4):904-8

47. Solerte SB, D'Addio F, Trevisan R, et al. Sitagliptin treatment at the time of hospitalization was associated with reduced mortality in patients with type 2 diabetes and COVID-19: a multicenter, case-control, retrospective. Observational Study. Diabetes Care. 2020;43(12):2999-3006.

48. Deeks JJ, Higgins JPT, Altman DG; the Cochrane Statistical Methods Group. Cochrane Handbook for Systematic Reviews of interventions. Version 6.1 , 2020. Chapter 10: Analysing data and undertaking metaanalyses. Specify characteristics in advance. https://training.cochrane.org/ handbook/current/chapter-10\#section-10-11-4. Accessed 30 July 2021

\section{Publisher's Note}

Springer Nature remains neutral with regard to jurisdictional claims in published maps and institutional affiliations. 